\title{
Feasibility of Household Surveys for Population Risk Assessment of Cancer and Cancer Registration Support
}

\author{
Omran S Habib ${ }^{1,2 *}$, Riyadh Abdul-Ameer Hussain ${ }^{1}$
}

\begin{abstract}
Cancer is a major health problem in the Arab region including Iraq. An adequate database is essential for effective cancer control strategies. Such a database may be provided through cancer registration but supportive household surveys may be useful. This article reports selected results on the feasibility of household surveys to support and validate cancer registration in Basrah governorate - southern Iraq. A large scale multi-stage cluster sample household survey was carried out in Basrah during 2013. It covered 6,999 households and involved gathering data on demographic characteristics and both incident cancer cases and cancer-related deaths among members of these households during a three-year recall period (2010-2012). The data obtained yielded an average annual incidence rate of 91 per 100,000 population (age-standardized incidence rate of $148.8 / 100,000$ ) and cancer specific mortality rate of 68 per 100,000 population (age-standardized mortality rate of 126.3/100,000). The results showed an overall pattern of cancer similar to that reported according to cancer registration but the household survey results were consistently higher than those of the cancer registration by a margin of approximately $20-30 \%$ with respect to incident cancer and about $70 \%$ with respect to cancer-specific mortality. Household surveys on cancer, while costly and time consuming, are a very useful additional source of information on cancer at the population level. They can be performed for specific purposes with effective resource mobilization.
\end{abstract}

Keywords: Cancer burden - epidemiology - risk assessment - household survey

Asian Pac J Cancer Prev, 17, Cancer Control in Western Asia Special Issue, 213-218

\section{Introduction}

In Iraq and particularly in Basrah in the southern part of the country, cancer has definitely increased in absolute numbers of new cases and numbers of deaths resulting from cancer. It is also believed with some empirical evidence that the incidence rates and mortality rates have increased. This is documented at least in a number of recent studies in Basrah (BCRG., 2010, Hagopian et al., 2010). A seven-year effort (2005-2011) made by the Basrah Cancer Research Group (BCRG) resulted in registering a total of 17080 new cases regardless of the place of residence. Of these, 11,679 (68.4\%) were from the inhabitants of Basrah governorate, the rest were from adjacent governorates seeking care in Basrah. Five cancers (Breast cancer, lymphomas (Hodgkin's and Non-Hodgkin's), urinary bladder cancer, lung cancer and leukaemias) are the leading incident cancers over the years 2005-2011 and comprise 45\% of all new registered cases in Basrah governorate. Other important cancers include those of skin $(4.1 \%)$, central nervous system $(4.1 \%)$, colon-rectum $(3.4 \%)$, Stomach $(3.1 \%)$ bones $(2.8 \%)$, Uterus-cervix $(2.3 \%)$ and larynx $(2.3 \%)$ (Habib and
Al-Ali., 2011). At present time, a strategy is adopted in Iraq by the Iraqi Cancer Board. The strategy consists of six approaches: population based registration, prevention, effective curative care through the enhancement of early detection and adequate amenities for treatment, palliative care for difficult and advancing cases, scientific research and voluntary work (Habib., 2005).

One of the most important requirements for effective control strategy is the availability of flow of relevant epidemiological data. The current sources of cancer related data are routine cancer registries (Iraqi Cancer Board., 2010), some limited household surveys (Habib et al., 2010, Naseef., 2012, Fashal et al., 2012) and limited research. Scientifically speaking, these sources would not give an adequate picture about the extent of the problem despite all the efforts involved in recent years to improve population-based cancer registration in Basrah and other governorates. A need was envisaged to validate the official cancer registration in Basrah by a number of means. One of these is a large scale household survey which is the subject of this paper. The survey aimed at a number of objectives, one of them is to test the feasibility of household survey to measure the risk of cancer and another is to validate cancer 
registration in Basrah. In this article we present detailed methodology adopted and few results of the survey.

\section{Material and Methods}

The reference population for the present study is all the population normally resident in Basrah governorate during the time of the survey. Basrah governorate is one of the 18 Iraqi governorates and is situated in the southern part of the country. It is bound by ThiQar and Missan governorates from the north, Iran from the east, Persian Gulf and Kuwait from the south and Kuwait and Al Muthanna governorate from the west. The total surface area of Basrah governorate is $19,070 \mathrm{~km} 2$ and an estimated population in 2014 at 2 676822 . The governorate is the main port of Iraq and is characterized by diversity of economic activities such as agriculture, industry, fishing and trading. Oil industry is a major component of economic activity.

The study is a cross-sectional survey with retrospective component to inquire about the incidence of cancer and cancer-related deaths during the three years preceding the date of inquiry. A convenient sample of 8,000 households with expected 48000 inhabitants was planned. A multi-stage cluster sampling approach was adopted: The sampling process is summarized below:

No. of health sectors 8 (All selected)

No. of primary health centres 100 (84 selected)

Households (estimated) 433,141 (6,999 selected)

The catchment population of each PHC center was already divided into clusters of houses (3-12 clusters) for purposes related to managing the immunization program and tracing defaulters. These clusters were assigned numbers in each PHC center and 1-2 housing clusters (50 households each) were randomly selected from the catchment population of each PHCC. The actual number of households visited and successfully interviewed was 6,999 households with a total 40,684 persons living in them. In each cluster a random starting point was agreed upon with the interviewers and from this point a circular sample is taken until around $(50 \pm$ few $)$ houses are consecutively visited.

A questionnaire form was preparedin the light of a thorough literature review (Damman et al., 2009, Anonymous, 2007), previous household -based research in southern Iraq (Habib et al., 2010, Naseef., 2012, Fashal

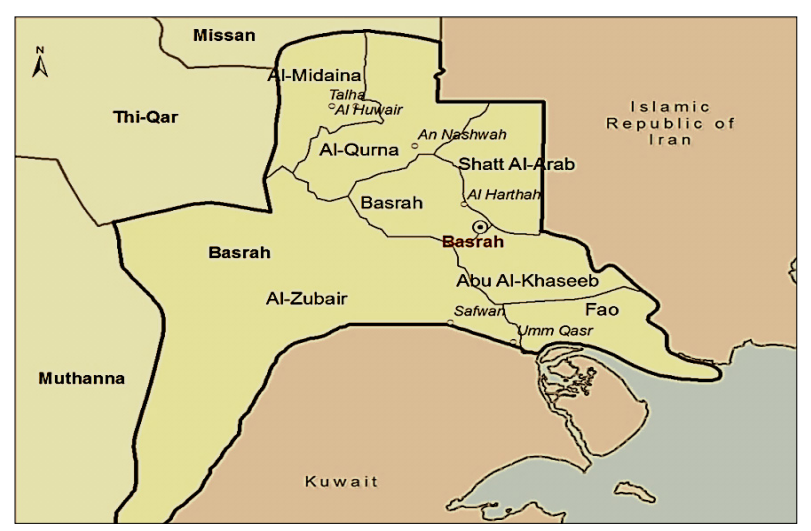

Figure 1. A Sketch Map of Basrah Governorate-Southern Iraq. et al., 2012) and guided by the study objectives. The questionnaire consisted of five sections:

Section One: Household characteristics.

Section Two: individual demographic characteristics.

Section Three: information on incident cancer to quantify the risk of cancer at population level.

Section Four: Mortality experience of the population during the three year-recall period including cancer-specific mortality.

Section Five: Data on profile of patient behavior in response to illness. This part is not further discussed in this paper.

The data were collected through direct interview of adult respondent from inhabitants of each house listed in the sampling frame.

The interviewers: The interviewers were trained teams selected from local health facilities and population in each of the eight health sectors in Basrah governorate. None of them was a medical doctor. Two steps were undertaken before the data collection phase. First, full explanation and instructions to each team on details of the study were made. Second, a pilot study was done on 100 households by each of the four teams employed to test the feasibility of using the questionnaire and identify unplanned problems. In addition, the questionnaire was discussed with senior staff at Basrah College of Medicine. Some modifications were made in the light of all these activities. At the end of every week, filled forms were collected from interviewers and grossly checked for errors.

Variables: A comprehensive list of variables was included in the questionnaire:

Household/Family: These two terms were used for the same concept in this study. One or more persons who live in one physical housing unit blood related to each other share, and pool their income and daily expenditure (Habib and Vaughan., 1986, Population Reference bureau., 2014).

A new case of cancer (incident cancer): A definite diagnosis of any type of cancer as reported by the interviewees and confirmed whenever possible by medical documents.

Residence area: As it is numbered sequentially from 1 to 84 . It was used as part of the sampling process and for sampling management and not for statistical analysis of the results.

Health sectors: Numbered from 1 to 8 . Also used for

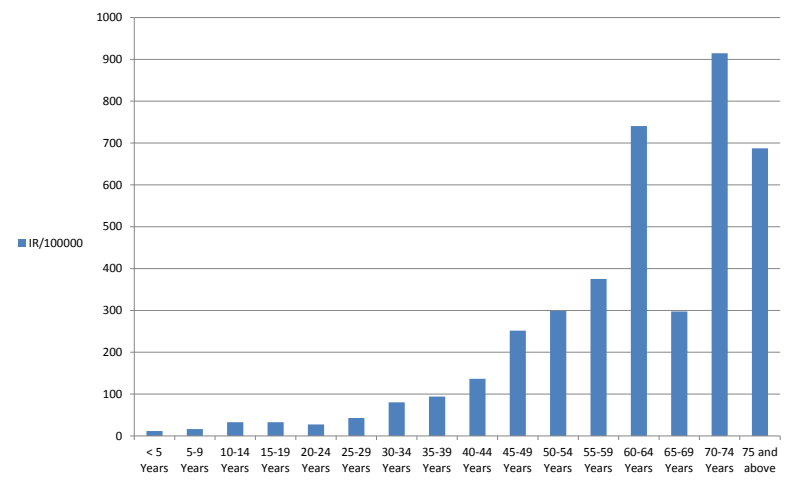

Figure 2. Age Specific Incidence Rate per 100,000 population: All Cancers According to the Household Survey Results 


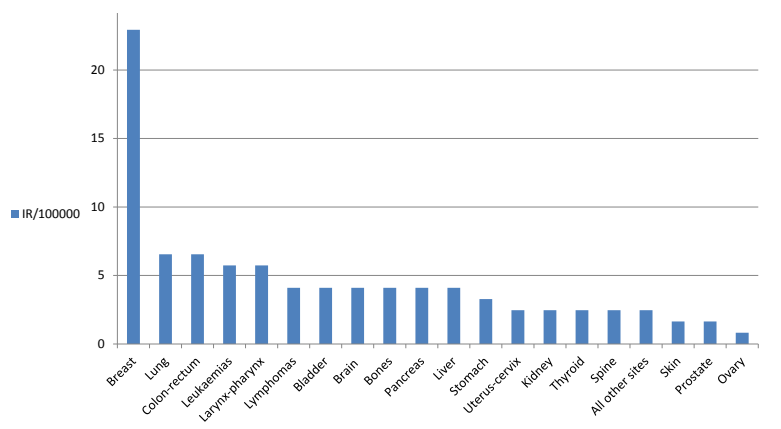

Figure 3. Types of Cancer Identified During the Household Survey: Incidence Rate per 100,000 Population.

sampling and management purposes.

Primary health care centers: Numbered from 1 to 100 in addition to writing their names.

Quarter/Village: named and numbered according to normal local nomenclature.

Household number: serial numbering of houses visited and included in the data collection process from 0,001 to 6,999 .

House ownership: As an indicator of socioeconomic status and coded as:

1. Owned, 2. Rented,3.Governmental and 4. Others

Type of building material of the house: 1. Bricks/ blocks, 2. Mud and 3. Reeds

Total monthly family income: The amount of money in Iraqi Dinars as reported by the family: Written as reported.

Total family numbers: The number of persons who normally lived in the house and considered as family members both from blood and economic point of view.

Total number of rooms in the house: This included all confined spaces in the house other than the kitchen and bathroom.

Per capita income: The average amount of money per person per month and equals the result of dividing the total monthly income of the family by the number of family members.

Crowding index: A measure of living conditions and was calculated as the average number of persons per room (No of persons in the family divided by No. of rooms in the house)

Car ownership: The number of cars owned by the family members.

Closeness of the house from risk imposing sources: This refers to any of the following if the latter is located within 100 meters from the house;

1. Bombed sites, 2. Scrub, war remnants, 3.Oil refinery or fuel selling station, 4.Power station, 5.Main traffic roads, 6 . Communication towers and 7 . Others

Age: Completed age last birthday in years as reported by respondents and confirmed by documents whenever possible. For the purpose of analysis, age was divided into 16 5-year groups:

Sex: male and female

Education: This refers to the highest level of education attained by the person.

Occupation: This represents the main type of job a person was engaged in at the time of interview, occurrence

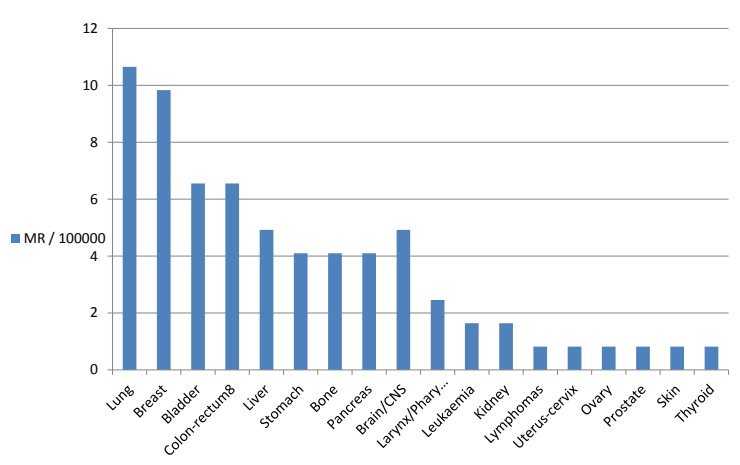

Figure 4. Cancer-Specific Mortality Rate per 100,000 Population According to Household Survey Results

of disease or death.

Marital status: Persons were divided according to their marital status at the time of interview into children less than 10 years old, unmarried among 10 +years old, currently married among 10+ years old, divorced and widowed.

History of onset of certain non-communicable diseases: Persons were inquired about any major non-communicable disease that has developed/recognized/ diagnosed during the three-year recall period. Inquiry was restricted to few NCD (Cardiovascular diseases, Cancer, Diabetes, hypertension)

Regarding any new cancer case during the three-year recall period, age, sex, type of cancer, date of diagnosis, and fate of the patient were obtained.

Regarding cancer related deaths during the three year recall period, age, sex, cause of death and year of death were ascertained.

Quality control: This included training and regular meetings with interviewers, periodic checking of filled forms and small sample cross-checking. In addition data were fed on computer programmes and checked for consistency and eligibility before the final analysis.

Ethical consideration: The data collection was confidential. Any family who needed any medical help were advised to the best choice possible. The research protocol was approved by the Scientific Committee at the Department of Community Medicine and the Ethical Committee for Research in Basrah College of Medicine.

Official endorsement: The study was approved by the following bodies:

1. The scientific Committee at the Department of Community Medicine.

2. The Committee on Research Ethics at the College of Medicine, University of Basrah.

3. The Research Committee at Basrah Health Directorate.

4. The College Council of Basrah Medical College.

Statistical analysis: Data were fed onto computer program using two types of media. First, the data were fed on an excel file as a data-base from which they were then transferred to an SPSS file (Statistical Package for Social Sciences-version 17). Intensive efforts were made to ensure accurate feeding of data and objectivity of analysis. Multiple files were made on subcomponents of the study. Data were presented in the form of tables and graphs as 
required. Because of the descriptive nature of the data, only occasionally a statistical significant tests were used.

\section{Results}

\section{Incidence by age}

Cancer incidence increases with advancing age as seen in Figure 2. A clear high incidence rates are seen for people in age groups 60 and above.

\section{Topography}

Cancer by type results are shown in Figure 3. Breast cancer is by far the most incident cancer in Basrah, followed by cancer of lung, colon-rectum cancer, leaukaemia and larynx-pharynx cancer. Other important cancers are lymphomas, urinary bladder, brain, bones, pancreas and liver.

\section{Mortality}

The two main cancer related deaths are attributed to lung and breast cancer followed by cancers of bladder, colon-rectum, liver, stomach, bone, pancreas and brain as shown in Figure 4.

\section{Discussion}

\section{Rational and adequacy of the methods}

\section{Is it justifiable to create a database for cancer?}

Cancer is the second (or third) leading cause of death in Iraq and Basrah and one of the main health problems in Basrah in terms of increasing incidence and mortality in addition to suffering of cancer patients as a result of delayed detection of disease and inappropriate with or without inadequate management measures. Hence cancer is considered as a political problem in addition to being a growing health problem. Therefore, it is necessary to put provincial plan to contain the cancer burden by decreasing the incidence and mortality with improving the quality of patient life. Such policy is totally in accordance with the national (Iraqi plan), regional (EMRO programme) and international moves (WHO 2014).

Cancer is amenable to effective control measures. At least one third of cancer cases could be prevented and another third could be detected early and treated with high degree of cure and last third of patients could be helped to improve their quality of life. Therefore, comprehensive cancer control approach should be one of the high priorities in the national plans for improving health status of our population. Quantification of cancer burden and survival at the population level definitely contributes to efforts of cancer control strategy. In other words, the presence of multiple sources of sufficient information about the risk of cancer occurrence, cancer mortality rates and distributions of cases according to age, sex, place and time are mandatory for establishing an effective Control measures of cancer (IAREC 2014). Cancer data can be obtained through different ways and the most important one is population-based cancer registrations (WHO 1999). No planning for cancer health care is effective without smooth flow of relevant information (Stewart and Wild,
2014). This is the strongest force behind the present study. It was intended to provide sufficient evidence on the status of cancer and cancer registration and clear the way towards adequate care planning and responsive cancer control measures.

\section{Adequacy of the sample}

Household surveys are essential components of any control programmes of health problems including the problem of major non-communicable diseases (NCDs), which are cardiovascular, cancer, diabetes and chronic obstructive respiratory diseases. The household surveys are used to gather information on risk factors, morbidity and mortality and health system responsiveness (WHO 2013). In cancer-data generation process, household surveys are not commonly used because of the fact that cancer is still a rare condition and a substantially large sample size is needed to obtain useful data. In the present study, a balance was made between the minimum but as reasonable as possible sample size studied and the assurance of the best representativeness of the sample to the reference population. The use of a three year recall period instead of one year recall period was meant for two purposes: first to improve sample size in theory instead of studying 6999 households for one year we studied their experience for three years (hypothetical tripling the sample size). Second to overcome the problem associated with short recall period (one year is considered short in the context of cancer).

\section{Representativeness of the sample}

The sample covered in the present study in the strict sense represents itself. Under the best of circumstances it represents the immediate blocks of houses from which it was drawn. Generalization beyond that must be considered with caution. However, given the fact that the sample spanned almost all residential areas in Basrah governorate and guided with the distribution of primary health centers, is a source of confidence that the sample is fairly representative of the population of the governorate in the general demographic, socioeconomic and risk exposure and health experience. What supports this view is the fact that certain indicators obtained in the study agree with published indicators like for example the age and sex structure of the population. Figures are very close to publish figures by the Iraqi Ministry of Planning and Developmental Cooperation (CSO, 2008, CSO, 2011, CSO 2013, UNESXCO 2010). Also the high degree of correspondence between characteristics of cancer patients obtained in the survey and those documented in Cancer Registry in Basrah is another indicator of the household sample fair representativeness of the general population.

\section{Possible sources of bias}

Bias, defined as deviation of results or inferences from the truth, or processes leading to such deviation cannot be ruled out completely in research on human beings particularly if data collection involves recall, memory and behavior. A serious case of bias occurs when a systematic error involves the different stages of research process; 
the design of the study, the data collection, analysis and interpretation (Manuel and Chris, 2004).

In the present study, avenues for bias can be suspected, given the complexity of the subject, the huge work at various stages and the human tendency towards certain preoccupations (Manuel and Chris,2004).

Many types of bias in epidemiology have been identified, but, for simplicity, they can be grouped into two major types: selection bias and measurement bias plus confounders though the latter are not biases in the scientific sense (WHO 1999).

Selection bias, which is a study design problem, occurs when randomness in choosing people for the study is not adhered to. This non-randomness may then distort the pattern of association between exposure and outcome (Manuel and Chris,2004). In the present study, Selection bias is minimal. The households/families have been completely selected randomly at least at the stage of clusters assignment. In addition, the sample was drawn from almost all areas in Basrah in order to avoid all sorts of bias related to selection of families in terms of those related to environmental characteristics, socioeconomic variations, or quality of health care facilities and accessibility to use these facilities. The latter affect risk of exposure, risk of disease and probability of contacting the health care sources and hence documentation of events. The sample involved the catchment areas of all eight sectors of primary health care in Basrah and then we took the catchments areas of the main primary health care centers of each sector. To avoid or at least minimize targeted households by interviewers (who may tend to search for known families with cases) strict instructions were given to the interviewers to move according the sampling plan put by the researchers.

Measurement (or information) bias occurs when measurements or classifications of disease or exposure are not valid (i.e., when the methods used fail to distinguish between diseased and non-diseased individuals). Errors in measurement may be introduced as a result of interviewer bias, (responder bias) or by the instruments inherited in the nature of the questionnaire content and questions to make the measurements (WHO 1999).

In our study, this type of bias could have occurred from both interviewers and respondents and nobody can claim complete avoidance of unplanned errors. We took a number of measures to minimize measurement errors. In case of interviewers, they were trained before conducting, met every now and then to respond to any queries and clarifications. A pilot study to assess the validity of questionnaire and the skills of interviewer to minimize the bias as possible as we could was done on 1,000 households and all necessary cross checking and amendments were made. Bias related to respondent recall cannot be totally excluded, but by aiding of respondent relatives (if needed) and asking the family for any hospital and other medical documents we might have minimized the effects of such bias. Also cancer incident event and death, both were the main theme of the study, are painful events. They are unlikely to be forgotten and very unlikely to be reported when they did not exist. Some kind of misclassification in certain cancer diagnoses or causes of death could have occurred but these must be small enough to distort the results of the study.

\section{Value of Household survey}

Population-based cancer registries are the main source of information on cancer incidence and mortality, particularly in developed countries with sufficient health care systems. Hence, they are the cornerstone for national cancer control strategies. While in low income countries where health care facilities are scarce, cancer registry activities are expected to be limited both in accuracy and coverage. Household surveys are good alternative sources for epidemiological data in general and for the cancer data as well (Hiat., 2002, Arozullah et al., 2004, Godard ., 2006, Costanzo et al., 2009). In Iraq and Basrah as well, with current scarce health care services in terms of limited resources and restricted rules, population-based registries are limited to be dependable for cancer control planning. Therefore, household survey is of great value to be complementary tool for the data reported by Basrah registration center, thereby cancer control activities could be planned and conducted effectively (Al-Hilfi and Habib., 2015, Al-Hilfy and Habib., 2015).

\section{References}

Al-Hilfi RA, Habib OS (2015). Incidence of cancer in Basrah: A household survey results. Asian Pac J Cancer Prev, 16, 163-7.

Al-Hilfi RA, Habib OS (2015). Cancer mortality in Basrah: A household survey results. Med J Basrah university, 33, 6-10.

Arozullah AM, Calhoun EA, Wolf M, et al (2004). The Financial burden of cancer: estimates From a study of insured women with Breast cancer. J Support Oncol, 2, 271-8.

BCRG (2010). Cancer in Basrah: epidemiological analysis of incident cancer 2005-2008. Basrah Cancer Research Group. Dar AlKutub for Press and Publication, Basrah.

Costanzo ES, Ryff CD, Singer BH (2009). Psychosocial adjustment among cancer survivors: findings from a National Survey of Health and Well-Being. J Health Psychol, 28, $147-56$

CSO (2008). Central Organization for Statistics and Information Technology (COSIT) of Iraq, the Kurdistan Region StatisticsOrganization (KRSO), and the International Bank for Reconstruction and Development/The World Bank. Iraq Household socio-economic survey -IHSES - 2007. Baghdad.

CSO (2011). Iraqi Ministry of Planning. Central Statistical Organization. Iraq Women Integrated Social and Health Survey (I-WISH).

CSO (2013). Iraqi Ministry of Planning. Central Statistical Organization. Statistic of Men and Women. Iraq.

Damman OC, Hendriks M, Sixma HJ (2009). Towards more patient-centred healthcare: a new Consumer Quality Index instrument to assess patients' experiences with breast care. Eur J Cancer, 45, 1569-77

Fashal MS, Habib OS, Abdul-Aziz T (2012). A comparative study on cancer in two areas in Samawa city. A confidential Report prepared for the Iraqi Cancer Board.

Habib OS, Al-Ali JK (2011). Cancer in Basrah 20052011: Characteristics of person, place and time. A confidential Report submitted to the Iraqi Cancer Board 2011.

Habib OS (2005). Environment and health in Southern Iraq: Facts and future prospects. Mesopot J, 20, 7-19.

Habib OS, Al-Ali JK, Al-Saad SL, Tooma JB, Wartanian K 
(2009). Cancer in Basrah:A population - based pilot household survey. A paper presented at the 1stInternational Oncology Conference in Basrah. Basrah May 7-9.

Habib OS, Vaughan JP (1986). The determinants of health services utilization in Southern Iraq. Int J Epidemiol, 15, 395-403.

Hagopian A, Lafta R, Hassan JG, et al (2010). Trends in childhood leukemia in Basrah, Iraq 1993-2007. Am J Public Health, 100, 1081-7.

Hiatt RA (2002). Cancer Screening Practices From National Health Interview Surveys. Past, Present, and Future. J Natl Cancer Inst, 94, 1837-46.

Iraqi cancer board (2010). Cancer Registry of Iraq. Ministry of Health, Peoples Medical Clinics Press, Baghdad.

Manuel B, Chris O (2004). Observational studies and bias in epidemiology. The young epidemiology scholars programme. College entrance examination board. 2004 pp 15. Available at:www.collegeboard.com. Accessed October 2014.

Naseef HK. Cancer in Hay Al-Khalij (2012). A household survey. Unpublished MSc thesis.

Population Reference Bureau (2014). Glossary of demographic terms. Available at: http://www.prb.org/Publications/LessonPlans/Glossary.aspx. accessed September 2014.

Stanford Patient Education Research Center. Stanford University School of Medicine. Sample questionnaire. August 2007.

Stewart BW, Wild CP. World cancer Report. 2014. IARC. Lyon, France. 2014

UNESCO (2010). Iraqi National Commission for UNESCO-Ministry of Education. Literacy Initiative for Empowerment in Iraq 2010-2015.UNESCO Iraq Office.

WHO (2014) NCD Global Monitoring Framework. http:// www.who.int/nmh/global_monitoring_framework/en/. Accessed December 2014.

WHO (1991) International Agency for Research on Cancer. Cancer epidemiology, principles and methods. Lyon, France.

WHO (2013). Global plan for the prevention and control of non-communicable diseases 2013-2029.WHO Press, World Health Organization, Geneva. 